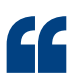

DNA damage at telomeres is irreparable

\title{
$\Rightarrow$ TELOMERES
}

\section{The perils of peripheral damage}

Cellular senescence - when cells can no longer divide - can be triggered by a prolonged DNA damage response (DDR). The combination of two studies has now revealed that DNA damage at telomeres is irreparable and that persistent damage foci trigger cellular senescence.

Fumagalli et al. and Hewitt et al. set out to define the nature of the damage foci that accumulate in senescent cells. They reasoned that telomeres, which are coated with factors such as telomeric repeat-binding factor 2 (TRF2) to prevent fusions between chromosome ends, might also suppress local

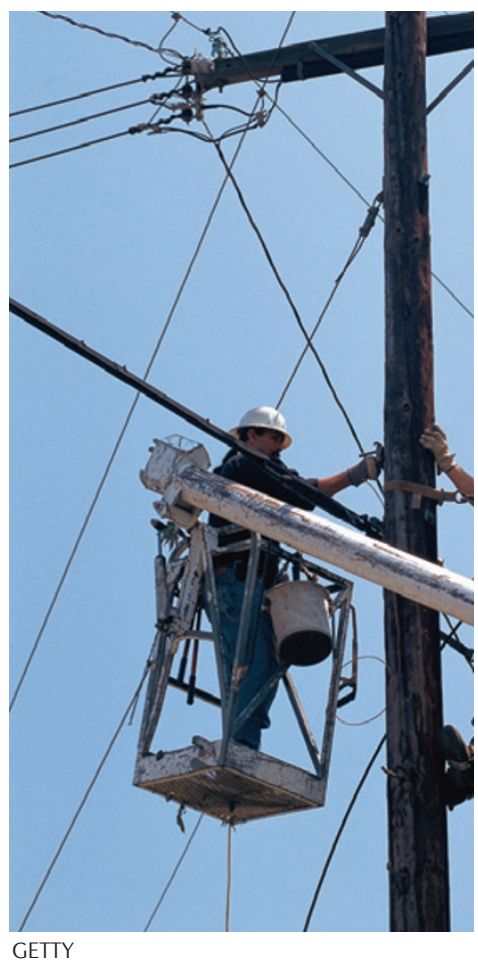

DNA damage repair. Fumagalli et al. first confirmed that exposing human fibroblasts to ionizing radiation produces damage foci that remain for months after cells have entered senescence and despite normal repair kinetics in these cells. Using immunofluorescence and immunoFISH (fluorescence in situ hybridization) with antibodies against the damage marker 53BP1 and against telomeric DNA tracts, respectively, they showed that the fraction of foci that persists at telomeres increases significantly with time. Similarly, Hewitt et al. showed that persistent foci accumulate at telomeres in human and mouse embryonic fibroblasts exposed to ionizing radiation and that these foci are insensitive to telomerase. Hewitt et al. also used live-cell microscopy to track the kinetics of telomeric versus nontelomeric DNA damage foci and found that the telomeric foci are longer lived. Using chromatin immunoprecipitation (ChIP) assays and different sequencing approaches, both groups showed that these persistent foci physically associate with telomeres. In addition, Fumagalli et al. found that whole-body ionizing radiation of mice triggered the formation of persistent telomeric foci in hippocampal neurons. Thus, exogenously induced DNA damage at telomeres cannot be repaired despite ongoing DDR signalling.

Fumagalli et al. went on to demonstrate that, when a double-strand break (DSB) is induced next to telomeric repeats in Saccharomyces cerevisiae, the DSB is no longer repaired.
Moreover, overexpression of a TRF2 fusion protein targeted next to an induced DSB in mouse fibroblasts also disrupts repair in cis and allows the DSB to persist. They also showed that microinjection of linear telomeric DNA (but not circular DNA) into cells triggers a DNA damage-induced checkpoint and cell cycle arrest.

DNA damage has been observed at telomeres of ageing primates, but it is unclear whether this might be driven by telomere shortening. Both groups showed that persistent foci are also observed at telomeres in tissues of ageing mice and baboons and, importantly, that this does not correlate with the presence of shortened telomeres. The authors propose that endogenous DNA damage also causes the formation of persistent foci at telomeres and thereby cellular senescence.

Although telomeres have evolved mechanisms to protect chromosome ends against fusions, an inherent consequence of this may be that any DNA damage incurred here cannot be resolved and ultimately triggers cells to enter senescence. This protects the organism against chromosomal instability, but also has implications for ageing.

Alison Schuldt

ORIGINAL RESEARCH PAPERS Fumagalli, M. et al. Telomeric DNA damage is irreparable and causes persistent DNA-damage-response activation. Nature Cell Biol. 18 Mar 2012 (doi:10.1038/ ncb2466) | Hewitt, G. et al. Telomeres are favoured targets of a persistent DNA damage response in ageing and stress-induced senescence. Nature Comm. 28 Feb 2012 (doi:10.1038/ncomms1708) 